\title{
An effective industrial control approach
}

\author{
Michal Kostoláni \\ Faculty of Electrical Engineering \\ and Information Technology \\ Slovak University of Technology \\ in Bratislava \\ Bratislava, Slovak Republic \\ Email: michal.kostolani@stuba.sk
}

\author{
Justín Murín \\ Faculty of Electrical Engineering \\ and Information Technology \\ Slovak University of Technology \\ in Bratislava \\ Bratislava, Slovak Republic \\ Email: justin.murin@stuba.sk
}

\author{
Štefan Kozák \\ Faculty of Informatics \\ Pan-European University \\ Bratislava, Slovak Republic \\ Email: \\ Stefan.kozak@paneurouni.com
}

\begin{abstract}
Requirements of increased productivity and flexibility in manufacturing processes reflect the concept Industry 4.0. Essential to achieving these targets is the implementation of intelligent and robust distributed control systems focused on interoperability and scalability. New approaches and technologies based on the Industrial Internet of Things (IIoT), cloud computing and Big Data are an emerging field of industrial control that comprises Internet-enabled cyber-physical devices with the ability to link new smart technologies. With this perspective, manufacturing devices can be easily monitored, operated and controlled even from remote locations. Conventional industrial control approach, that use programmable logic controller (PLC), is enhanced with intelligent industrial gateway IoT 2040 as a hardware and Node-RED software environment in order to provide interoperability, robust and reliable control system. Collected process data and parameters from embedded sensors and other connected devices can be quickly collected, processed, transformed and used for device control from remote production environment. This paper deals with an industrial gateway framework adopting the idea of Internet of Things for the development of robust industrial control approach. The concept is tested in real industrial environment.
\end{abstract}

\section{INTRODUCTION}

$\mathbf{l}_{\mathrm{i}}$ NDUSTRY 4.0 represents the fourth industrial revolution in manufacturing industry with complex automation, cyber-physical systems, data exchange and Industrial Internet of Things (IIoT) principles. The ability of integration and cooperation of intelligent machines, methods and human beings to interact is essential condition for increased productivity and flexibility in manufacturing processes [1].

Interconnection of machines, embedded sensors, digital devices and people continues to extend. The aims of this approach are improved industrial manufacturing processes, efficient ways to operate production plants, services and supervision for industrial installations, reduced operational cost in relation to requirements of improved human safety. The IIoT offers interconnection and intelligence to industrial systems and machines through sensing devices and actuators with ubiquitous networking and computer abilities [1], [2].

The expectation toward industrial applications related to intelligent hardware, software and serviceability are high.
Nowadays, many IoT academic research studies, publications and applications related to the IIoT principles are being developed. The development of intelligent control prototypes has increased mainly due to educational development kits, such as Rasberry Pi, Arduino, etc., that are usually not certified for the use in industry.

The difference between industrial hardware and regular development kit are in UL/ CE certification that indicates conformity with health, safety, and environmental protection standards for industrial continuous operation applications. Secure installation within machine and electrical panels allows minimal industrial IP 20 housing and easy connection with other DIN rail industrial devices, such as relays, power supplies or PLC. For continuous operation in industrial environment must be this hardware built with industrial grade components resistant to vibrations, dust, high temperature and electromagnetic interferences. Regarding communication with other industrial hardware, it must be possible by using secure industrial protocols, such as Profinet, MQTT or ModBus [2].

Developing an effective industrial control approach and IIoT application according to the IIoT principles requires meeting the following requirements on increased [3]:

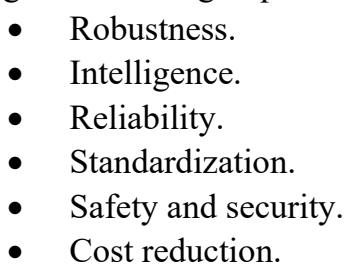

The architecture of conventional industrial control system consists of distributed embedded devices, such as PLC that control physical processes and supervisory computer that gather data and command PLC. Modern industrial control systems are distributed, offer higher performance and often connected to the internet or intranet [1], [4].

Machines from different manufactures and on different technological levels often do not use the same communication protocols or programming language. In order to satisfy the need for intelligent and robust control solution suitable for industrial production that harmonizes communication between the various data sources, the 
architecture of industrial control system must be enhanced with an industrial certified gateway [4].

IIoT gateway builds the bridge between sensors and actuators of manufacturing device on one hand, and the internet or intranet on the other hand. IIoT gateway moreover comprises other capabilities such as filtering the amount of data or security implementation as depicted in Fig.1.

Industrial gateway represents the reliable open platform for collecting, processing and transferring data in the production environment. It is intelligent gateway between a company's IT department and industrial solutions and it is important to note that the role as an interface can be used in both directions.

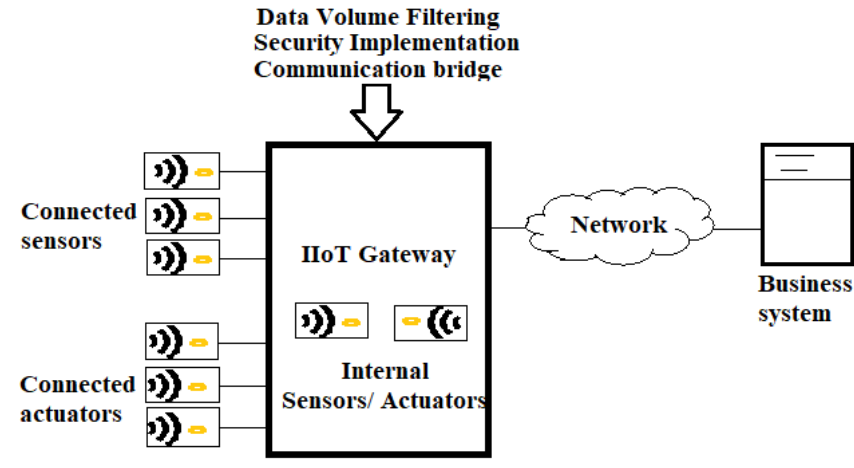

Fig. 1 Industrial IIoT gateway function principle

The paper consists of four parts. In the first part requirements for effective industrial control according to the IIoT methodology are defined. The second part deals with the problem formulation of industrial control systems current state and intelligent gateway. The third part of the paper provides a description of the proposed approach and system implementation in terms of hardware (IoT 2040) and software components (Node RED). The fourth part deals with case study - verification of proposed approach.

\section{PROBLEM FORMULATION}

This paper proposes a robust, effective and modern approach of process control and remote monitoring system of a real physical model represented by an automated working system in compliance with the main IIoT requirements, interoperability and scalability [4]. This standalone system is depicted in Fig.2 and represents an autonomous working station of production line with sorting mechanism and consists of industry certified hardware, such as power supply, engine with frequency inverter, conveyor belt, proximity sensors, pneumatic actuators and programmable control unit. The main functionality of this system is to move packages along the conveyor belt to the sorting mechanism, which divides packages according to the shapes into individual containers. Control of actuators and monitoring of process parameters in manual or automatic operation is only possible by touch panel or control buttons.

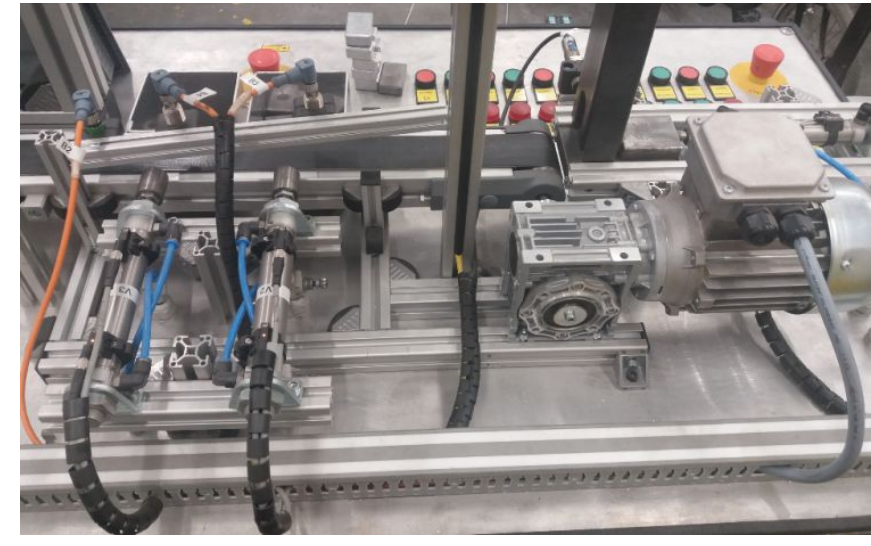

Fig.2 Autonomous stand-alone working station of production line

If there is a system error or breakdown, the operator has to check all process conditions, quit error messages and start the automatic operation through the touch panel.

If there is one system, it is a simple task. If the system is more complex or without the operator's permanent control, there is no possibility to control and improve production processes of the system.

As it is a stand-alone system without access to any network, intranet or internet, interoperability with cloud based systems, other cyber-physical systems or remote monitoring system is not possible. To improve interconnection, scalability, time and cost savings, it is necessary to enhance the stand-alone system with external hardware which enables the connection of working station with a local network [4].

As the best candidate for solving this problem in industrial conditions, Simatic IoT 2040 shown in Fig. 3 has been chosen as the open industrial gateway [6]. IoT 2040 is an open platform for collecting, processing and transferring the data between production and IT systems or clouds in the production environment. It is designed for $24 / 7$ operation and the role as an intelligent gateway interface can be used in both directions transferring data. This gateway supports programming languages such as Java, Python or $\mathrm{C}++$, and multiple communications protocols such S7 Protocol, OPC UA, Profinet, TCP IP, MQTTL via various interfaces, including RS232/422/485, serial USB, Ethernet or Wi-Fi.

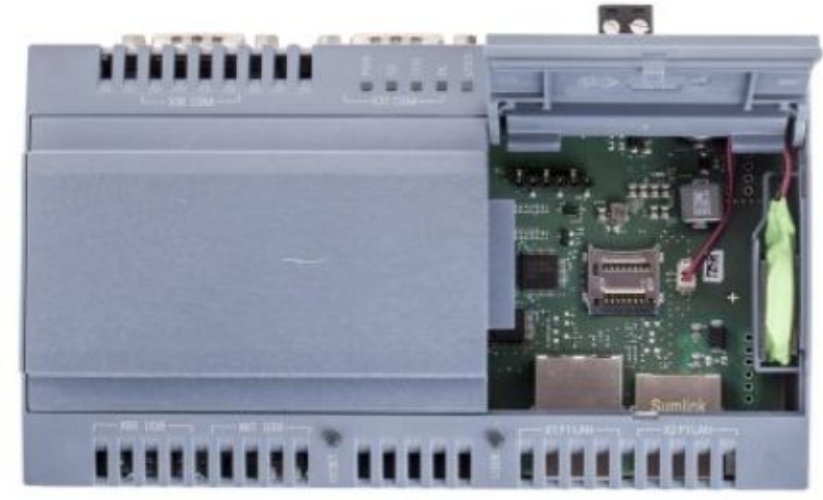

Fig. 3 Industrial gateway IoT 2040 


\section{SYSTEM IMPLEMENTATION}

Typical industrial control systems consist of distributed embedded devices such as PLC S7-1500 that control physical processes. For effective and robust industrial control approach according to the principles of IIoT, it is essential the hardware and software implementation and configuration of intelligent gateway as depicted in Fig. 4.

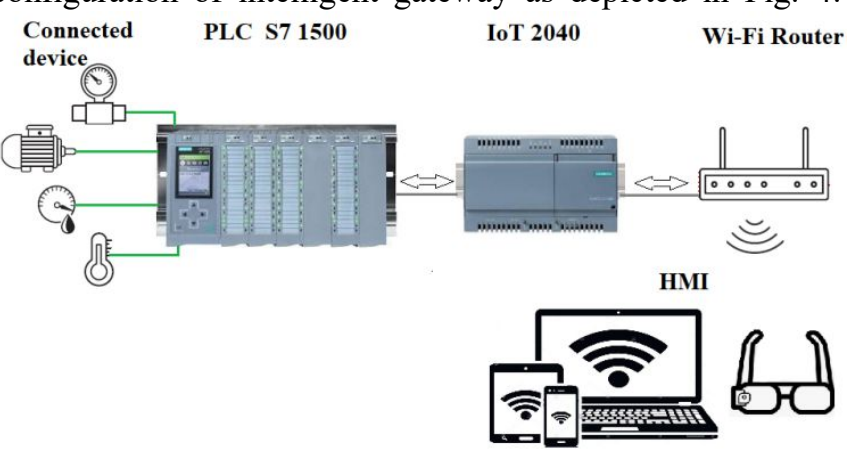

Fig. 4 Implementation of gateway IoT 2040 in to typical control system

In following parts, the exact approach of hardware and software implementation and configuration of IoT 2040 will be analyzed.

\section{A. Setting up S7-1500}

Based on environment and technical requirement, PLC S7-1500 was used for real-time control of the stand-alone system described above. Devices, such as sensors, actuators or frequency inverter are connected via Profinet cable directly to the I/O modules of PLC. Communication between these devices is possible via libraries in Totally Integrated Automation (TIA) software [4].

\section{B. Hardware implementation}

To the existing real physical model represented by automated working system controlled via PLC the intelligent gateway can be attached horizontally or vertically on a DIN rail or to a wall. Connection to the power supply can only be supported by 9-36 V. The Ethernet cable connected to the port X1 P1 LAN provides the gateway connection with WiFi router. Communication between IoT 2040 and PLC S71500 was ensured via Ethernet cable connected to the gateway port X2 P1 LAN as depicted in Fig. 5.

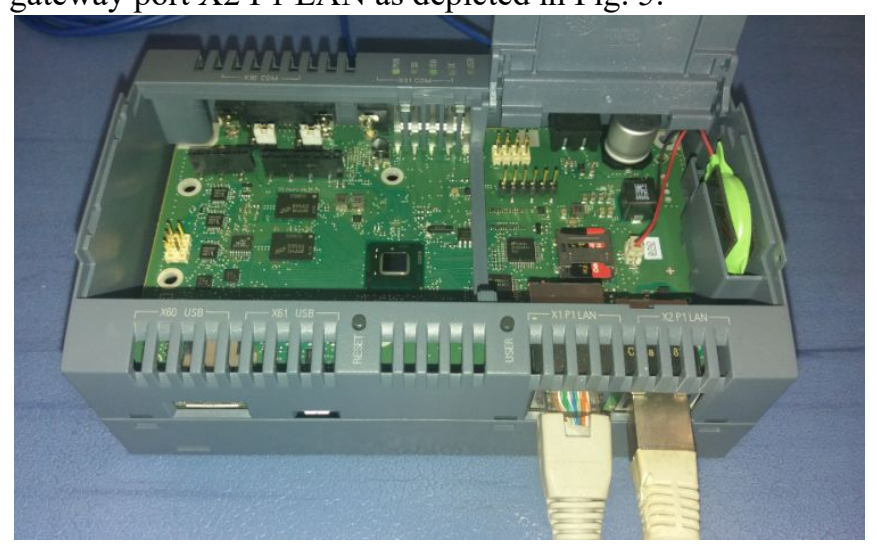

Fig. 5 Hardware implementation of industrial gateway IoT 2040

\section{Software implementation of PLC S7 1500}

The first step in software implementation of PLC S7 1500 and connected devices, such as sensors and actuators, is to set communication between PLC and I/O modules via device configuration.

Once the communication is established the variables type, address and tag table need to be defined. Networks with appropriate variables from a list are an essential condition for creating automated control system as shown in Fig. 6.

To allow communication with other devices connected to the network, the permission access with PUT/GET communication from remote partner, such as PLC, HMI or OPC must be enabled [7].

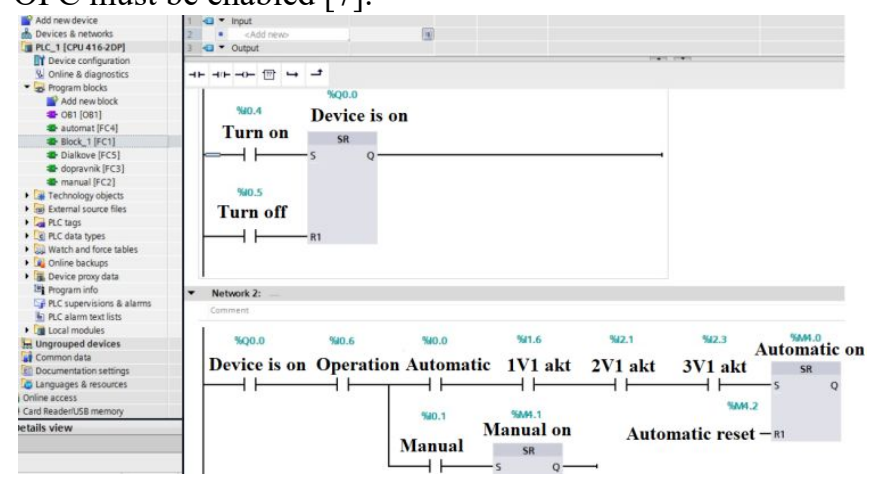

Fig.6 Setting of control program with the use of PLC S7 1500

\section{Software implementation of IoT 2040 in Node-RED}

Industrial gateway IoT 2040 is certified hardware for industrial application. According to the type of application, the image must be created and uploaded to the gateway [5]. The first step for working with industrial gateway IoT 2040 is to set up a micro-SD card with appropriate image and install it to the gateway.

To create IIoT application, the interconnection of the physical layer with Node-RED needs to be established. To start Node-RED, a special command must be typed into the gateway terminal. Once the Node-RED has started, the user interface can be open in the web interface after typing the correct IP address 192.168.43.200:1880 and logging in with user credentials. Basic settings such as IP address of PLC, port, rack, slot, cycle time must be defined to establish the interconnection between correct hardware components. In order to allow remote control and remote visualization of connected devices using gateway IoT 2040, in PLC defined variables need to be defined as well in Node-RED variable list. After completing of basic communication and variables settings, it is possible to continue with the creation of application flows.

The process of flow creation is similar to the PLC flowbased programming. To the input nodes that are located on the left side of Node-RED web editor it is appropriate to assign them in the workspace with a proper variable and other relevant settings [5]. The same logic is used to create output debug nodes. To establish the connection between input and output nodes the wire in web editor is used. 


\section{CASE STUDY}

The aim of this case study is to verify the proposed methodology on a diminished form of gearbox production line with sorting mechanism by which parts are divided into individual containers. Individual parts are stored in a magazine and are in regular intervals positioned on the moving conveyor belt. According to the evaluation of the sorting mechanism, individual components are then moved by means of pneumatic cylinder. A HMI (Human Machine Interface) is used as a touch panel. B1, B2, B3 and B4 represent sensors, V1, V2 and V3 represents pneumatic actuators. According to the above mentioned methodology of IIoT application, the data from PLC are used for remote control and visualization. To create a simple application, input nodes connected to the sensor variable (I0.4) and engine variable (Q1.2) are wired with different types of output nodes, for example pneumatic actuators (Q0.0), conveyor belt (M50.1) as depicted in Fig. 7.

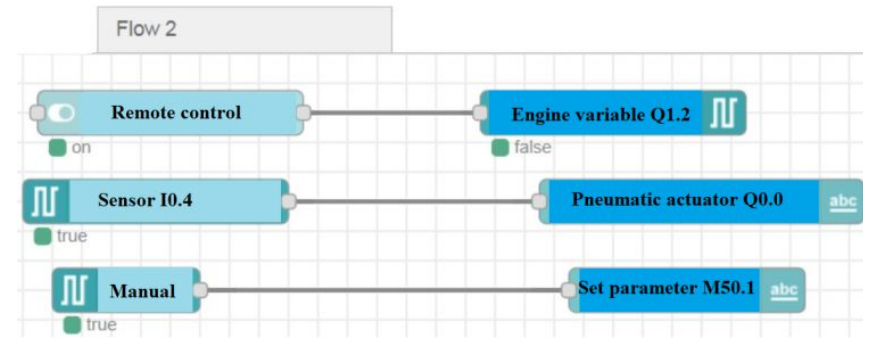

Fig. 7 Settings of input and output nodes in IIoT application in NodeRED

According to the specific requirements of different applications, output information from PLC can be presented in form such as gauge, switch, email, twitter, etc. After all nodes are created, the whole flow needs to be deployed.

Once the whole flow is deployed, remote visualization of the station and its connected components in real time is possible via any smart devices connected to the same local network after typing the correct IP address and log in using safety $\log$ in credentials. In the start page of the application, there is the option to set the device to automatic or manual operating mode, as well as to allow the remote control.

In the manual operating mode, using smart phone or other device connected into the same network, is it possible to see remote visualization of variables as depicted in Fig. 8.

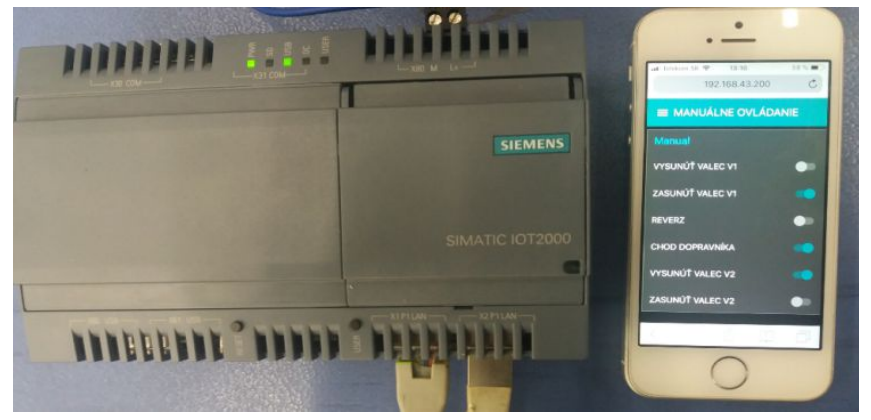

Fig. 8 Manual operating mode of mechatronic system via smart phone
To change variables defined in variable list and control the process by changing parameters on switch, gauge or conveyor belt in real time is possible even via smart phone.

\section{CONCLUSION}

In this paper, we managed to interconnect an industrial PLC via intelligent gateway IoT 2040 with web based platform Node-RED. Relevant data for remote control and visualization, such as current state of connected devices or process data can be easily obtained directly from PLC to intranet or internet without the need of additional sensor installation. A case study according to the proposed methodology was applied to verify this approach.

Using this approach, process data is easily accessible even from remote locations, can be analyzed and evaluated to improve production or maintenance processes. This scalable result is a solution that can be extended to the cloud based systems.

Further research and experiments should focus on applying of proposed scalable approach to the more complex industrial equipment for further possibilities of collecting, evaluating obtained data, as well as the use of secured cloud based systems in real time.

\section{ACKNOWLEDGMENT}

This article is supported by the Cultural and Educational Grant Agency of the Ministry of Education, Science, Research and Sport of the Slovak Republic, grant No. KEGA 030STU-4/2018 and Scientific Grant Agency of Ministry of Education, Science, Research and Sport of Slovak Republic, grant No. VEGA: 1/0102/18.

\section{REFERENCES}

[1] M. Wollschlaeger, T. Sauter, a J. Jasperneite, "The Future of Industrial Communication: Automation Networks in the Era of the Internet of Things and Industry 4.0", IEEE Industrial Electronics Magazine, roč. 11, č. 1, p. 17-27, mar. 2017. DOI: 10.1109/MIE.2017.2649104

[2] I. Zolotová, M. Bundzel, and T. Lojka, "Industry IoT Gateway for Cloud Connectivity", in Advances in Production Management Systems: Innovative Production Management Towards Sustainable Growth, 2015, s. 59-66. DOI: 10.1007/978-3-319-22759-7 7

[3] A. Astarloa, U. Bidarte, J. Jiménez, A. Zuloaga, and J. Lázaro, "Intelligent gateway for Industry 4.0-compliant production", in IECON 2016 - 42nd Annual Conference of the IEEE Industrial Electronics Society, 2016, p. 4902-4907. DOI: $10.1109 /$ IECON.2016.7793890

[4] A. Gavlas, J. Zwierzyna, a J. Koziorek, "Possibilities of transfer process data from PLC to Cloud platforms based on IoT", IFACPapersOnLine, roč. 51, č. 6, s. 156-161, jan. 2018. DOI: $10.1016 /$ j.ifacol.2018.07.146

[5] J. Skovranek, M. Pies, a R. Hajovsky, "Use of the IQRF and NodeRED technology for control and visualization in an IQMESH network", IFAC-PapersOnLine, roč. 51, č. 6, s. 295-300, jan. 2018. DOI: $10.1016 /$ j.ifacol.2018.07.169

[6] S. Toc a A. Korodi, "Modbus-OPC UA Wrapper Using Node-RED and IoT-2040 with Application in the Water Industry", in 2018 IEEE 16th International Symposium on Intelligent Systems and Informatics (SISY), 2018, s. 000099-000104. DOI: 10.1109/SISY.2018.8524749

[7] M. Kostolani, J. Murin, S. Kozak, "Intelligent predictive maintenance control using augmented reality" in $22^{\text {nd }}$ International Conference on Process Control, 2019, p. $131-135$. 